\title{
Grass cell wall feruloylation: distribution of bound ferulate and candidate gene expression in Brachypodium distachyon
}

\author{
Hugo B. C. Molinarit , Till K. Pellny, Jackie Freeman, Peter R. Shewry and Rowan A. C. Mitchell*
}

Plant Biology and Crop Science, Rothamsted Research, Harpenden, Hertfordshire, UK

\section{Edited by:}

Maurice Bosch, Institute of Biological, Environmental and Rural Sciences, Aberystwyth University, UK

\section{Reviewed by:}

Ana Winters, Aberystwyth University, UK

Carsten Rautengarten, Joint BioEnergy Institute, Lawrence Berkeley National Laboratory, USA Jaime Barros-Rios, Umeå University, Sweden

\section{${ }^{*}$ Correspondence:}

Rowan A. C. Mitchell, Plant Biology and Crop Science, Rothamsted

Research, Harpenden, Hertfordshire AL5 2JQ, UK.

e-mail: rowan.mitchell@rothamsted. ac.uk

${ }^{\dagger}$ Present address:

Hugo B. C. Molinari, Laboratory of Genetics and Biotechnology, Embrapa Agroenergia, Brasilia, Federal District 70770-901, Brazil.
The cell walls of grasses such as wheat, maize, rice, and sugar cane, contain large amounts of ferulate that is ester-linked to the cell wall polysaccharide glucuronoarabinoxylan (GAX). This ferulate is considered to limit the digestibility of polysaccharide in grass biomass as it forms covalent linkages between polysaccharide and lignin components. Candidate genes within a grass-specific clade of the BAHD acyl-coA transferase superfamily have been identified as being responsible for the ester linkage of ferulate to GAX. Manipulation of these BAHD genes may therefore be a biotechnological target for increasing efficiency of conversion of grass biomass into biofuel. Here, we describe the expression of these candidate genes and amounts of bound ferulate from various tissues and developmental stages of the model grass Brachypodium distachyon. BAHD candidate transcripts and significant amounts of bound ferulate were present in every tissue and developmental stage. We hypothesize that BAHD candidate genes similar to the recently described Oryza sativa p-coumarate monolignol transferase (OsPMT) gene (PMT sub-clade) are principally responsible for the bound para-coumaric acid ( $p C A)$, and that other BAHD candidates (nonPMT sub-clade) are responsible for bound ferulic acid (FA). There were some similarities with between the ratio of expression non-PMT/PMT genes and the ratio of bound FA/pCA between tissue types, compatible with this hypothesis. However, much further work to modify BAHD genes in grasses and to characterize the heterologously expressed proteins is required to demonstrate their function.

Keywords: glucuronoarabinoxylan, hydroxycinnamic acid, BAHD gene family, PF02458 domain, bound phenolic

\section{INTRODUCTION}

Many of the most abundant potential lignocellulosic feedstocks are from grasses, whether by-products from food crops such as wheat straw, rice straw, maize stover, and sugar cane residues or from specialized bioenergy crops such as Miscanthus and switch grass. Such feedstocks differ from those from dicotyledonous crops in the occurrence of trans-ferulic acid (FA), a hydroxycinnamic acid which is ester-linked to the hemicellulosic component, glucuronoarabinoxylan (GAX; Carpita, 1996; Scheller and Ulvskov, 2010). This linkage occurs only in commelinid monocots and is particularly abundant in grasses (Harris and Trethewey, 2010). It is considered to affect digestibility (Grabber, 2005; Dhugga, 2007), as FA can oxidatively cross-link to form ether bonds or C-C bonds, linking chains of GAX (Ishii, 1991), or of GAX to lignin (Ralph et al., 1995). Thus it covalently links the cell wall polysaccharide which can be used as a substrate for biofuel to the inhibitory lignin component. Whilst the barrier to degradation presented by FA is mainly structural, FA also has antimicrobial activity, inhibiting sugar fermentation by yeast (Baranowski et al., 1980; Akin, 2008). Grasses with decreased feruloylation of GAX in their cell walls may therefore be more readily converted into biofuel. In particular, they will have a lower energy requirement for separation of lignin from polysaccharide, an essential step in most pipelines for biomass processing.
The genes and enzymes responsible for feruloylation of GAX are still unknown. However, using a bioinformatics analysis of publicly available expressed sequence tags (ESTs), we identified an orthologous group within the BAHD acyl-coA superfamily as potential candidates based on their differential expression in cereals compared with dicots (Mitchell et al., 2007). The BAHD family (also referred to as the PF02458 family, as all family members contain the PFAM domain PF02458 which is specific to the family) is named after the first four members to be biochemically characterized (benzylalcohol acetyltransferase, BEAT; anthocyanin hydroxycinnamoyl transferase, AHCT; anthranilate hydroxycinnamoyl/benzoyl transferase, HCBT; deactylvindoline acetyltransferase, DAT; D'Auria, 2006). The predicted role in GAX feruloylation is compatible with the several hydroxycinnamoyl transferase activities creating ester bonds known in the family, with feruloyl-CoA acting as the donor. We also identified GT61 genes as likely to be involved in GAX biosynthesis (Mitchell et al., 2007), and have recently demonstrated (in collaboration with the Dupree lab, University of Cambridge, UK) that these genes mediate the addition of 3-linked arabinofuranose (Araf) to xylan (Anders et al., 2012). GT61 genes tend to be co-expressed with the BAHD candidates in cereals (Mitchell et al., 2007; Shewry et al., 2011), consistent with a role in feruloylation of the 3-linked Araf residues added by GT61 proteins. Our predicted role of BAHD 
genes in feruloylation was tested by another group using RNAi suppression in rice, showing a modest $(-19 \%)$ but significant decrease in cell wall bound ferulate in the stems of the transgenic plants (Piston et al., 2010). However, this effect could be an indirect consequence of gene suppression, so the role of these BAHD genes still requires elucidation.

One question is the intracellular localization of the proteins encoded by the BAHD genes: this is predicted to be cytosolic based on the protein sequences and this is the localization for all known members of the family (D'Auria, 2006), whereas GAX is synthesized in the Golgi (Buanafina, 2009). An activity from rice seedlings capable of feruloylation of Arafon a synthetic molecule was found in the soluble fraction rather than the membrane fraction, consistent with a cytosolic enzyme (Yoshida-Shimokawa et al., 2001). A possible explanation is that BAHD proteins are responsible for feruloylation of a cytosolic precursor, such as UDP-Araf, which is the substrate for xylan arabinosylation. Whereas the great majority of UDP-arabinopyranose is synthesized in the Golgi lumen, this is converted by a mutase to the UDP-Araf form in the cytosol (Konishi et al., 2007; Rautengarten et al., 2011) so this is a possible substrate for a cytosolic feruloylation reaction in planta. The product from this would then be transported back into the Golgi, where feruloylated Araf would be transferred onto GAX, possibly by a GT61 enzyme. The feruloylation of arabinoxylan has been shown to occur within the protoplast (Myton and Fry, 1994), although there is also evidence of an alternative pathway which allows feruloylation in the wall when inhibitors are used to suppress the secretory pathway (Mastrangelo et al., 2009).

A key recent breakthrough was the demonstration that at least one of these BAHD genes in rice has a different role from that predicted; in that the encoded protein catalyzed the addition not of FA to a sugar, but of the closely related para-coumaric acid ( $p$ CA) to monolignols. This gene therefore encodes a $p$ CA monolignol transferase and has been named OsPMT (Oryza sativa p-coumarate monolignol transferase; Withers et al., 2012). Interestingly, whereas other BAHD proteins have been shown to use both FA-CoA and pCA-CoA as donor molecules (Luo et al., 2007), OsPMT was found to have almost no activity with FA-CoA (Withers et al., 2012). Whereas it is theoretically possible that OsPMT could also add FA or pCA to Araf, activities with such different acceptors have not previously been found for BAHD proteins. OsPMT activity is presumably responsible for forming ester linkages between $p C A$ and lignin, a linkage which is also much more common in grasses than in other groups of plants. It is therefore possible that the candidate group which includes OsPMT is responsible only for this activity, rather than also for GAX feruloylation. However, to date, only one of the genes has been shown to have this activity and others are expressed (albeit at low levels) in wheat starchy endosperm which does not have lignin but does have feruloylated AX (Pellny et al., 2012), suggesting that other genes within the group are responsible for xylan feruloylation. During preparation of this article, strong support for this theory was published where another BAHD gene within this group was overexpressed in rice, resulting in increased bound $p \mathrm{CA}$ with good evidence that this was esterlinked to GAX, not lignin (Bartley et al., 2013). Whereas this gene seems specific for $p C A$, it seems very likely that some of the other similar genes in the clade are responsible for FA ester-linked to GAX.

Brachypodium distachyon is a model grass species which has a small and fully sequenced genome, a short life cycle, and small size, making it ideal for the study of grass cell walls (Vogel et al., 2010). We have therefore determined the contents of bound FA and $p$ CA (predominantly linked to GAX and lignin, respectively) in various tissues and at different stages throughout the life cycle, and related these to the expression of BAHD genes identified as candidates for the addition of these phenolic acids to cell wall polymers (GAX and lignin).

\section{RESULTS AND DISCUSSION}

Figure 1 shows a phylogenetic tree of the clades of the BAHD gene superfamily which have been proposed to contain candidate genes for the feruloylation of GAX in grass cell walls (Mitchell et al., 2007), divided into clades A and B. Clade B genes are not highly expressed or co-expressed with xylan pathway genes, and do not have clear orthologs between rice and Brachypodium. The more highly expressed clade A genes were therefore identified as the stronger candidates (Mitchell et al., 2007) for AX feruloylation. Clade A also includes the gene subsequently shown to encode a monolignol pCA transferase in rice, OsPMT (Withers et al., 2012). Two wheat genes, orthologous to candidates 1 and 3, are expressed in wheat starchy endosperm, which does not contain lignin in the cell walls (Pellny et al., 2012). An RNAi construct which simultaneously suppressed the expression of rice candidates $1,4,8$, and 10 (in our nomenclature) resulted in a decrease in cell wall FA of $19 \%$ in transgenic rice (Piston et al., 2010). A possible interpretation is therefore that the monolignol $p C A$ transferase activity is restricted to the sub-clade containing OsPMT which includes candidates 7 and 9 and the only Arabidopsis gene, and the other candidates in clade A may be involved in GAX feruloylation. This is supported by the recent demonstration that overexpressing OsAt10 (coincidentally also candidate 10 in our nomenclature) resulted in increased $p C A$ almost certainly ester-linked to GAX (Bartley et al., 2013). This ester linkage is less common than FA in most tissues and is absent in wheat starchy endosperm. From all the evidence, we hypothesize that the activity responsible for GAX feruloylation will exist in some or all of the candidates $1-5$ and 8.

The expression of candidate genes and amounts of bound FA and $p \mathrm{CA}$ were determined in tissues of developing $B$. distachyon plants as shown in Figure 2.

The transcript abundances of the BAHD genes (denoted by the candidate numbers shown in Figure 1), were determined by quantitative real-time RT-PCR (qRT-PCR) and the values are shown for the different genes, tissues, and developmental stages in Figure 3. The primer efficiencies were comparable for the different genes (Table 2), so differences in the abundances determined for the genes should reflect real differences in transcript abundances. On this basis, it can be concluded that the BAHD gene candidates which are most highly expressed in above-ground tissue samples from the vegetative phase are, in descending order of abundance, $5,9,1$, whereas in roots they are 1,9 . In later stages of reproductive development, they are $9,7,5$, and $2 \mathrm{p} 2$, and in the spike at the advanced stage 7, 10, 5 (Figure 3 ). Therefore, BAHD genes 


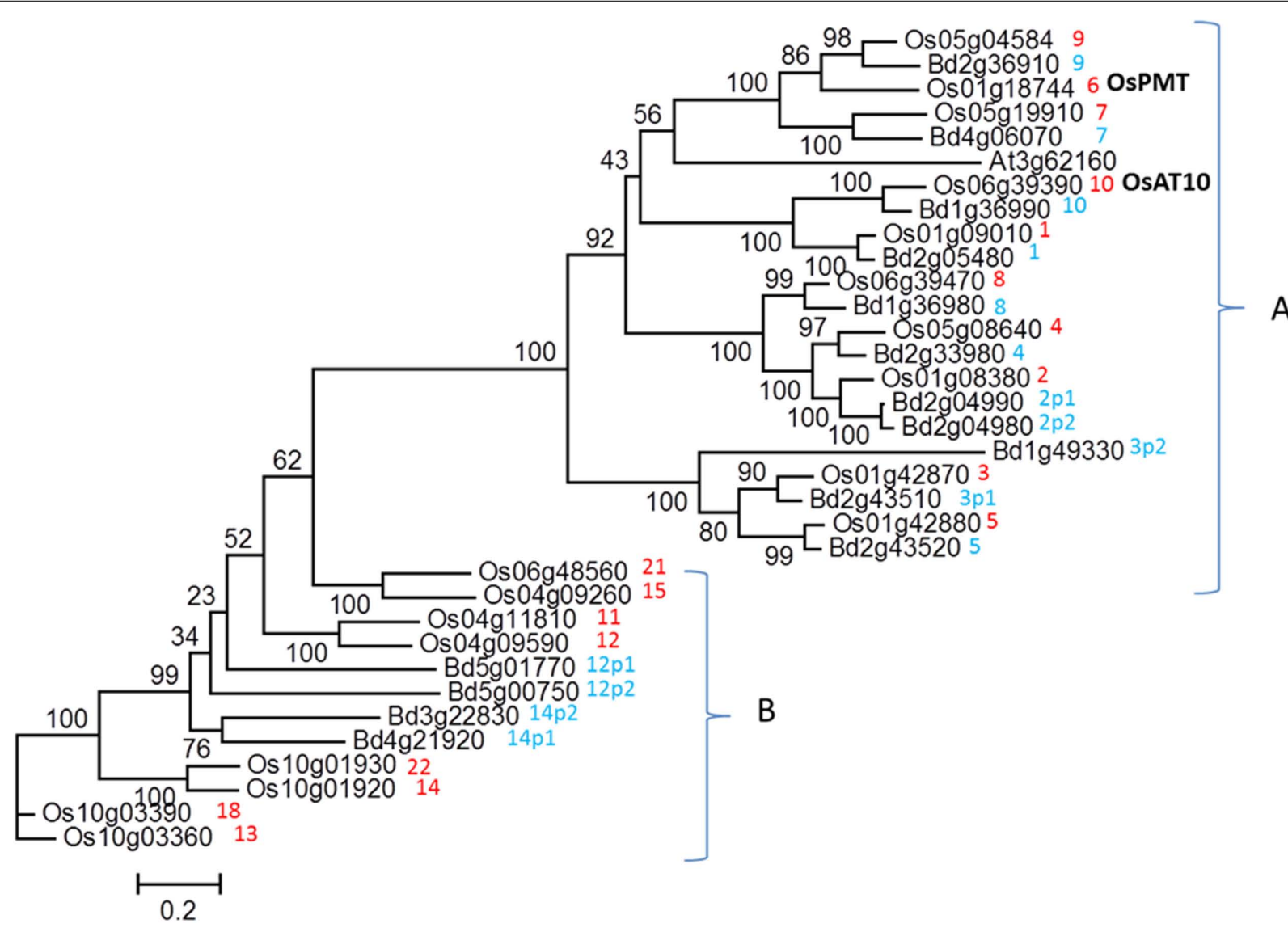

FIGURE 1 | Phylogenetic tree of clades within BAHD gene superfamily for rice and Brachypodium genes, which were identified as containing potential candidates for xylan feruloylation (Mitchell et al., 2007).

Numbers are arbitrary candidate numbers based on rice genes; where two paralogous Brachypodium genes match one rice gene these are given the same number but with "p1" or "p2" appended to the name. Genes within clade $\mathrm{A}$ are generally more highly expressed than those in clade $\mathrm{B}$. The recently identified monolignol pCA transferase OsPMT Withers et al., 2012) and OsAt10 (involved in pCA addition to xylan; Bartley et al., 2013) genes are indicated. from clade A are highly expressed in all tissues that were analyzed. Based on the phylogenetic relationship with OsPMT (Figure 1) and the assumption that BdBAHD 9 and 7 are responsible for the addition of coumaryl esters on lignin (i.e., PMT activity), and the others responsible for xylan feruloylation (with the exception of 10 which is more likely responsible for coumaryl esters on xylan), it can be noted that at least one gene with each function is highly expressed in every tissue that was analyzed, with the genes with PMT activity being relatively more highly expressed than other BAHD candidate genes in the reproductive growth phase.

Determination of amounts of bound phenolic acids in plant samples is a measure of the amounts that are covalently linked to the cell wall fraction. Protocols using moderate alkaline treatment release only the ester-linked phenolics from cell walls, which are then separated and quantified by high-performance liquid chromatography (HPLC). By far the most abundant bound phenolic acids are FA and $p$ CA. FA is considered to be exclusively linked to GAX by ester bonds in grass cell walls, while the pCA is predominantly ester-linked to lignin, with a much lower amount ester-linked to GAX. FA, unlike $p$ CA, can oxidatively couple in planta to form dimers or higher-order oligomers. The amounts of bound FA monomer, major FA dimers and bound $p$ CA are shown for different tissues in Figure 4. The amount of $p C A$ reflects principally the amount of this phenolic acid which is ester-linked to lignin, and this fraction has been found to be highly correlated with the degree of lignification in grasses (Grabber et al., 2004). The values shown are therefore consistent with increased lignification during the reproductive phase, relative to GAX feruloylation, which occurs in both primary and secondary grass cell walls. The absolute amounts of FA and pCA in young shoots (early vegetative phase, EVP) determined here are also comparable with those determined for seedlings at 8 days after germination (DAG) where FA and $p$ CA were found to be $\sim 400$ and $\sim 200 \mu \mathrm{g} \mathrm{g}^{-1}$, respectively (Christensen et al., 2010) and with a recent analysis of more tissues and stages (Rancour et al., 2012). A consistent feature of the data presented here (Figure 4) and by Rancour et al. (2012) is that the $p$ CA:FA ratio is higher in stems compared to leaves, which is consistent with the comparatively greater lignification in stems.

Expression of BAHD candidates from both the PMT and nonPMT sub-clades was found in every tissue examined (Figure 3), as was substantial quantities of bound FA and pCA (Figure 4). 

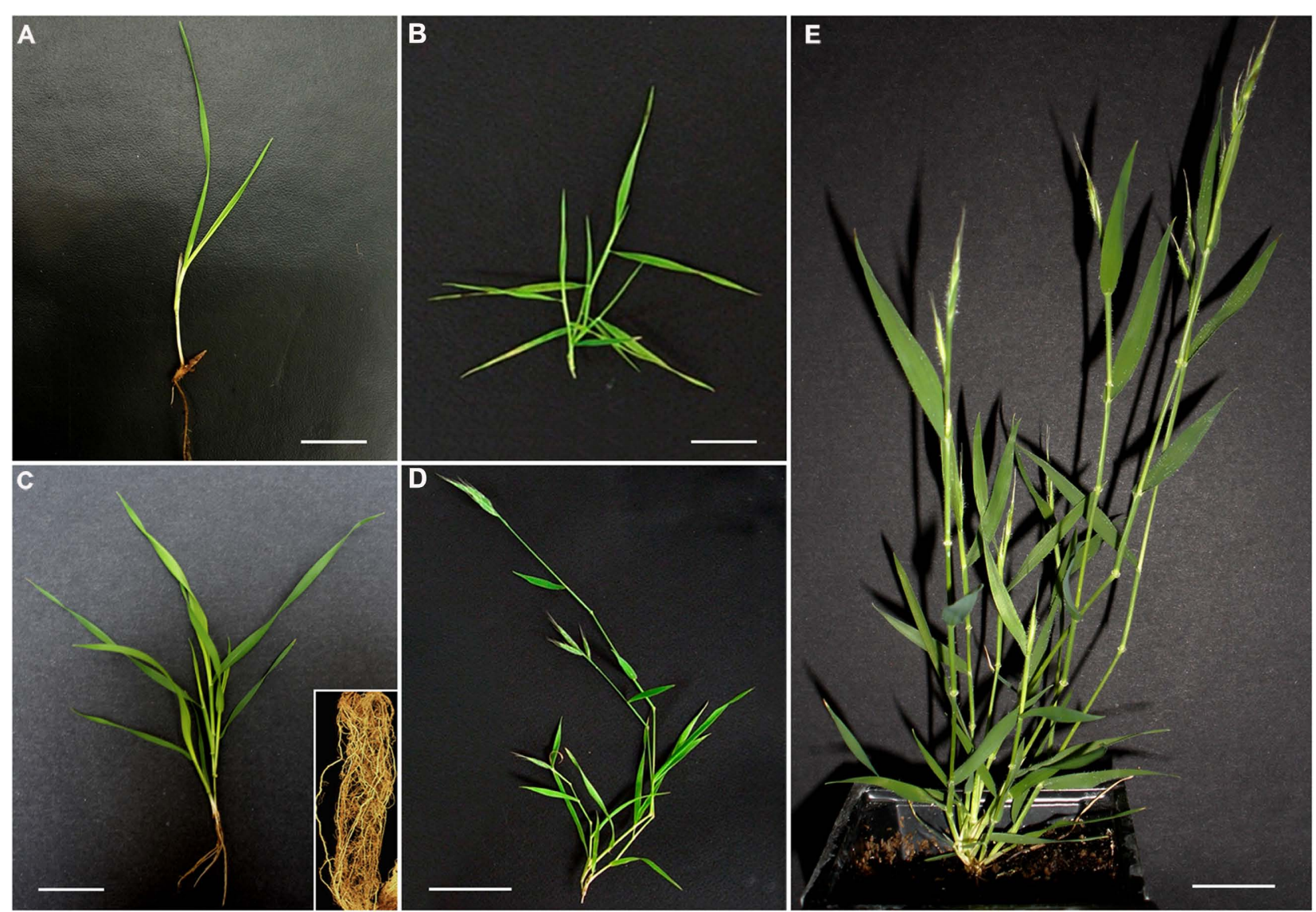

FIGURE 2 | Brachypodium developmental stages. (A) Early vegetative phase (EVP), bar = $1 \mathrm{~cm}$; (B) late vegetative phase (LVP), bar = $2 \mathrm{~cm}$; (C) transition phase (TP), bar $=2 \mathrm{~cm}$; (D) reproductive phase $(R P)$, bar $=3 \mathrm{~cm}$; (E) advanced phase (AP), bar $=2 \mathrm{~cm}$.

Simple correlations are not expected between the abundances of transcripts encoding enzymes in a biosynthetic pathway and the amount of end-product from that pathway. Transcript abundance measures the potential for enzyme synthesis only at a single time point whereas end-product accumulation occurs throughout a developmental process and may be limited by substrate availability as well as enzyme activity, which in turn depends on enzyme activation as well as enzyme amount. However, tissues which tend to have more bound FA compared to total bound pCA, might also be expected to have greater expression of BAHD candidates in the non-PMT sub-clade relative to those in the PMT sub-clade. We found this to be broadly the case, with shoots during vegetative phase and leaves in later phases, which have high bound total FA top $p$ CA content (due to less lignification), also having greater ratios of non-PMT to PMT expression than internodes, roots, and spike (Figure 5).

We have previously identified co-expression between BAHD candidates and xylan synthesis genes, compatible with a role in GAX feruloylation (Mitchell et al., 2007; Shewry et al., 2011), but given the new findings on these candidate genes (Withers et al., 2012; Bartley et al., 2013), we decided to re-examine this. Public resources are not yet extensive for Brachypodium gene expression, but using the RiceFREND tool which employs a wide range of rice transcriptome experiments (Sato et al., 2013), we examined co-expression for the rice BAHD genes with rice genes putatively involved in xylan synthesis (Table 1). Nearly all the clade A genes had xylan synthetic genes in the top $1 \%$ of genes ranked by expression correlation, but none of the clade B did. BAHD1, 5, and 8 showed particularly close co-expression with genes involved in UDP-Xyl and UDP-Ara synthesis and GT61 genes, whereas OsPMT and similar genes (BAHD7 and BAHD9) had fewer closely co-expressed genes. If these genes in the PMT sub-clade are responsible for addition of $p$ CA to lignin then some co-expression may be expected with any genes involved in secondary cell wall xylan synthesis.

\section{CONCLUSION}

The genes identified as candidates for being involved in feruloylation of GAX (Mitchell et al., 2007) are expressed in every tissue and at every developmental stage in the model grass Brachypodium as expected for a process which is required for every primary and secondary cell wall in the plant. The relative amounts of pCA and FA in these different tissues seem compatible with the expression of BAHD candidate genes, where these are divided into those putatively responsible for $p$ CA linked to lignin (PMT) and FA linked to xylan (non-PMT). The evidence presented here 


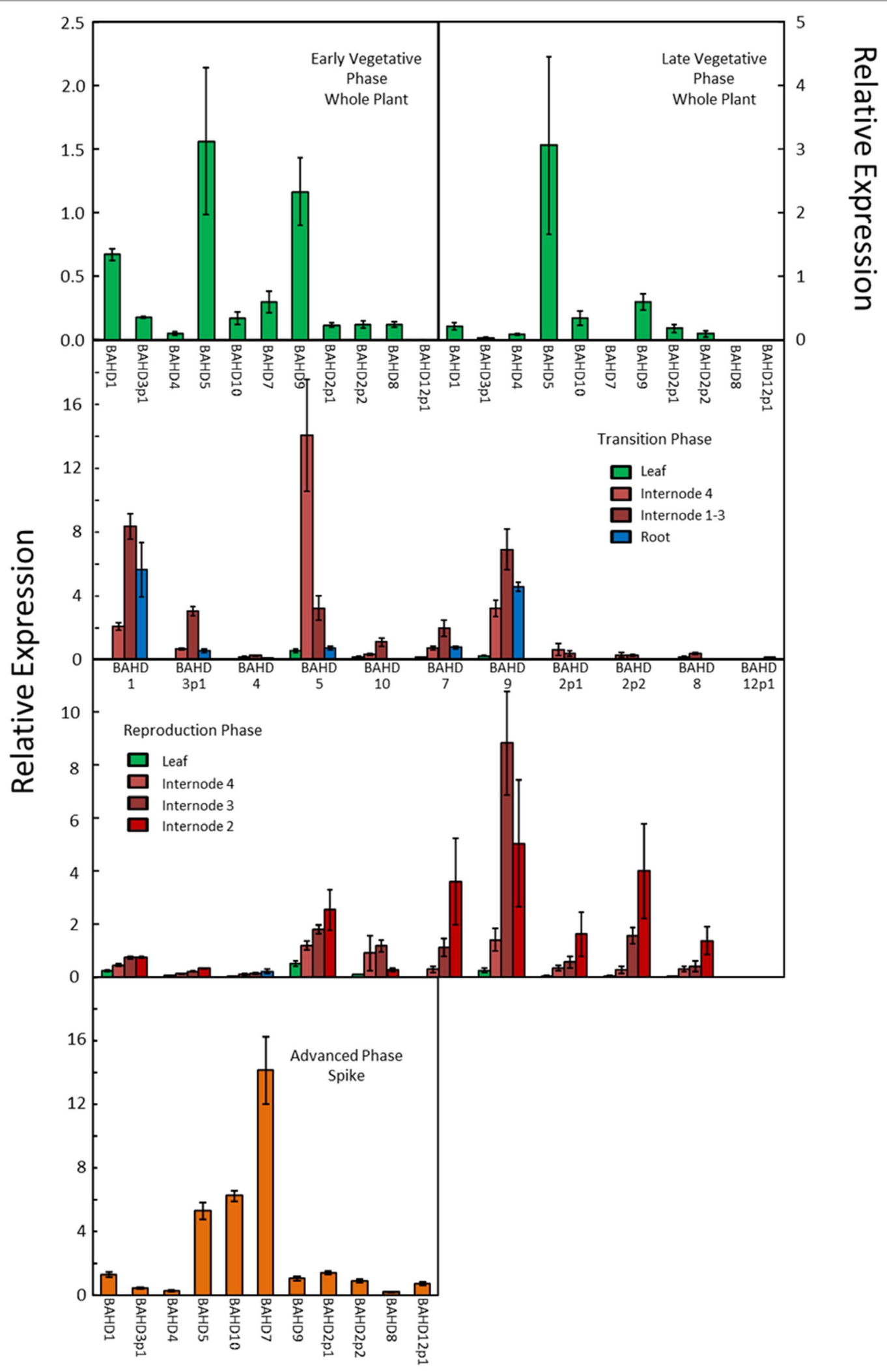

FIGURE 3 | Transcript abundance of candidate BAHD genes in developing Brachypodium tissues estimated by qRT-PCR. Error

bars are \pm SE from three biological replicates. One-way analysis of variance showed that the effect of tissue/stage on transcript abundance was significant at $F$ probability $<0.001$ for every gene except BAHD2p1, where it was significant at $F$ probability $=0.002$. 


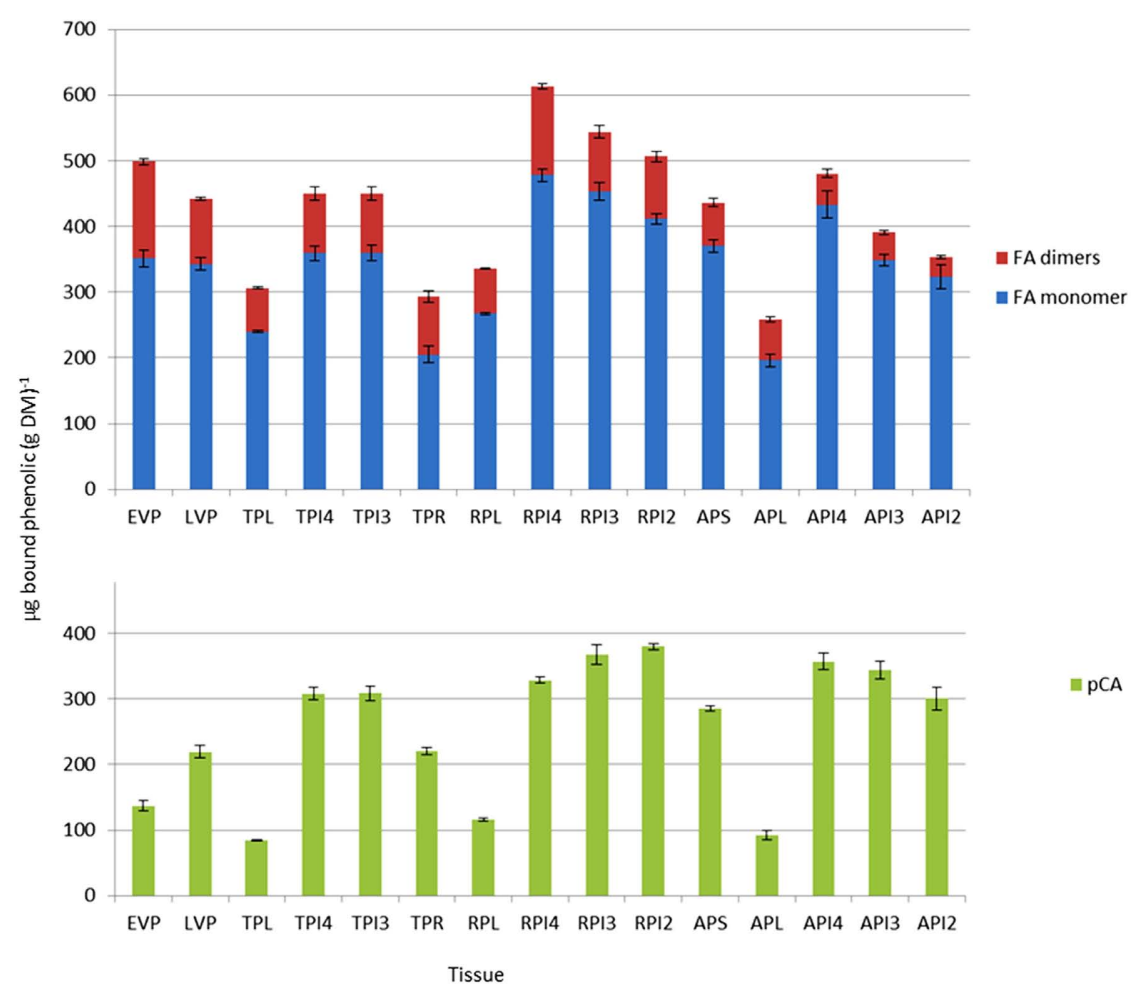

FIGURE 4 | Bound hydroxycinnamic acid content from developing Brachypodium tissues estimated by HPLC. Samples were early vegetative plants (EVP), late vegetative plants (LVP), transition phase leaves (TPL), transition phase internodes (TPI4, TPI3), transition phase roots (TPR), reproductive phase leaves (RPL), reproductive phase internodes (RPI4, RPI3, RPI2), advanced phase spike (APS), advanced phase leaves (APL), and advanced phase internodes (API4, API3, API2). FA dimers are the sum of the four major dimers described in Section "Materials and Methods." Error bars are \pm SE from three biological replicates. One-way analysis of variance showed that the effect of tissue/stage on both total FA and pCA content was significant at $F$ probability $<0.001$.

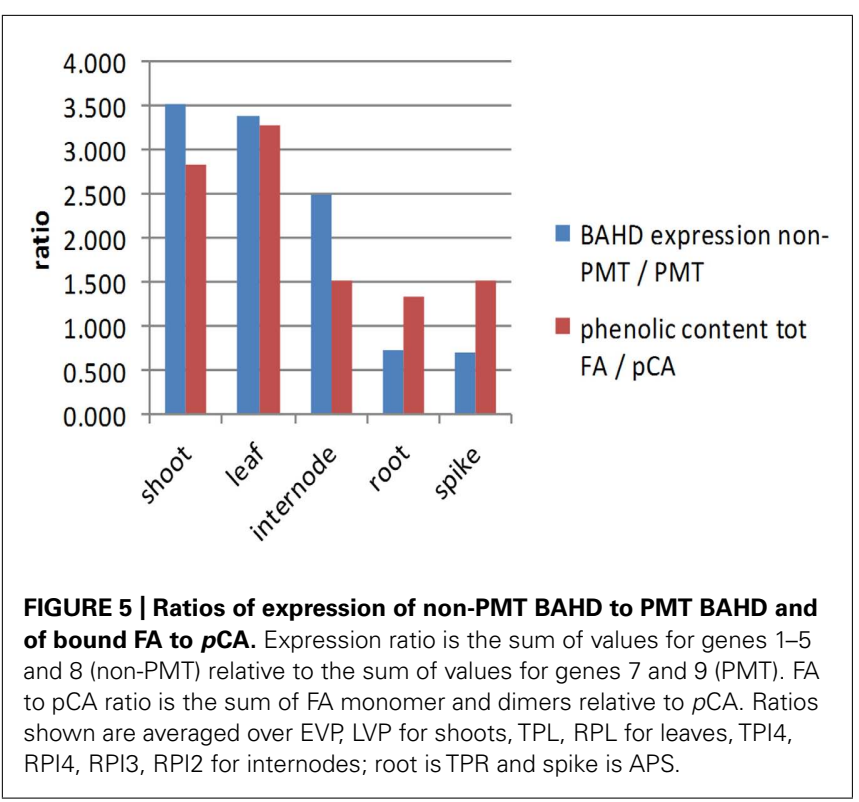

does not demonstrate function; for this more studies with targeted modification of the genes in grass species (as in Bartley et al., 2013) and in vitro characterization of pure proteins (as in
Withers et al., 2012) are required. If their role in feruloylation is confirmed by such experiments, then as feruloylation is believed to be key to the exploitation of grass biomass for biofuel and animal feed, these genes would represent important biotechnological targets.

\section{MATERIALS AND METHODS PLANT GROWTH}

Brachypodium plants $\mathrm{Bd} 21$ were grown in standard glass house conditions at $25^{\circ} \mathrm{C}$. The whole aerial plant was harvested for the EVP (7 DAG) and late vegetative phase (LVP; 12 DAG). Plants were vernalized at $4^{\circ} \mathrm{C}$ for 2 weeks during the EVP; references to "DAG" do not include this period. In the transition (20 DAG) and reproductive (30 DAG) phases, the plants were separated into leaf and internode tissues, whereby internode 1 is the oldest and 4 the upmost and youngest. In the transition phase, roots were also harvested. Spikelets, i.e., the developing seeds with the surrounding maternal tissues, were harvested in the advanced phase (50 DAG). Material was immediately frozen in liquid nitrogen and stored at $-80^{\circ} \mathrm{C}$.

\section{PHYLOGENETIC ANALYSIS}

Protein sequences for the whole BAHD superfamily were identified as all rice, Arabidopsis, and Brachypodium sequences from 


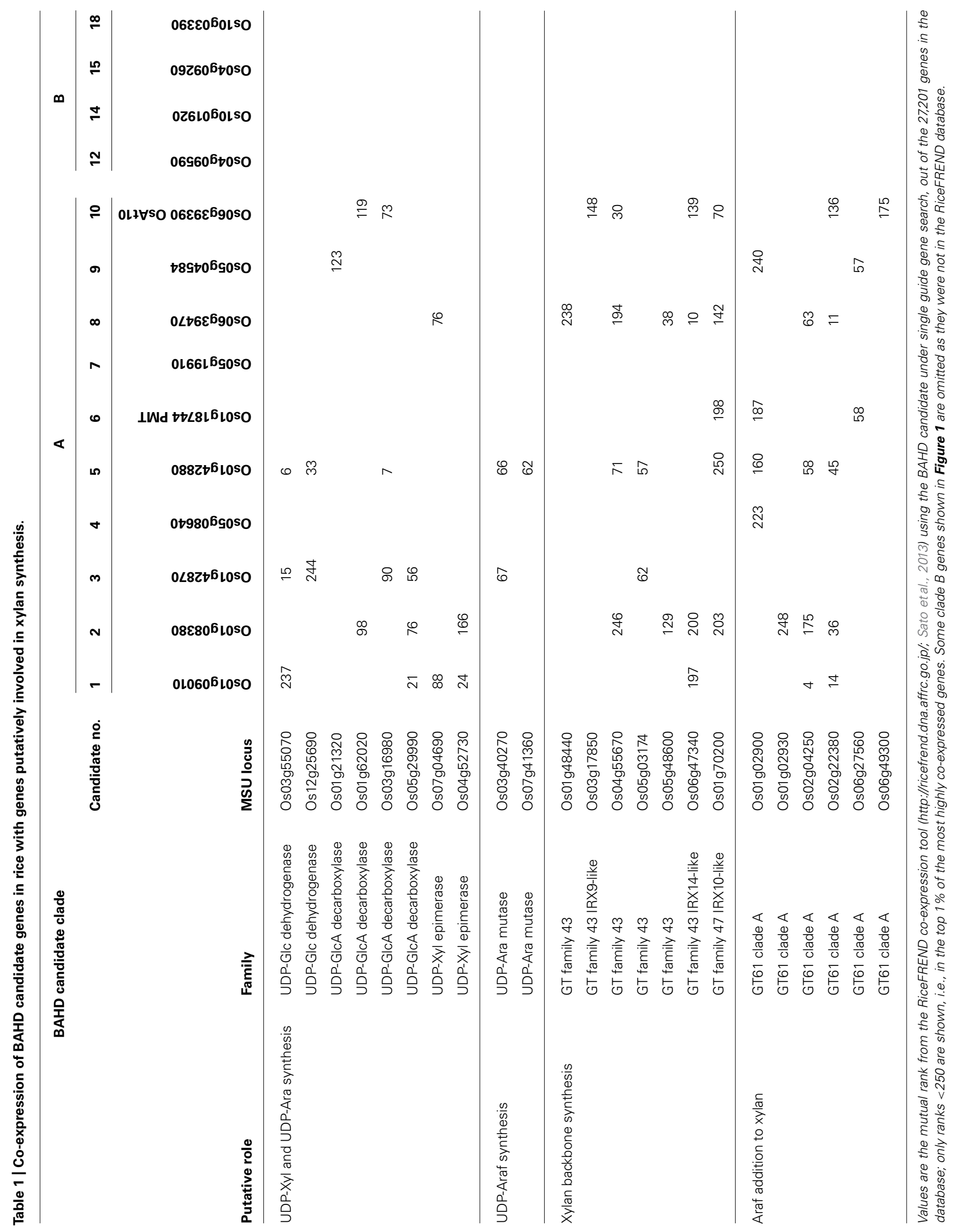


Table 2 | PCR primers.

\begin{tabular}{|c|c|c|c|c|}
\hline Oligo name & Orientation & Sequence $\left(5^{\prime}-3^{\prime}\right)$ & Target & Average primer efficiency \\
\hline prHM01 & Sense & GCCGCACAACACCATCATG & qRT-PCR Amplicon Bd_Bahd_1 & 1.74 \\
\hline prHM02 & Antisense & GGCTTTGATGAACTGCGCC & qRT-PCR Amplicon Bd_Bahd_1 & \\
\hline prHM07 & Sense & АССТСАTССTCATGGCCCA & qRT-PCR Amplicon Bd_Bahd_3p1 & 1.745 \\
\hline prHM08 & Antisense & CGAAAACCAGGTGGCTGAAG & qRT-PCR Amplicon Bd_Bahd_3p1 & \\
\hline prHMO9 & Sense & CCGGTAAGGTGGCCTCGT & qRT-PCR Amplicon Bd_Bahd_4 & 1.84 \\
\hline prHM10 & Antisense & CGAACTCTGAAGGCAGCCG & qRT-PCR Amplicon Bd_Bahd_4 & \\
\hline prHM11 & Sense & CGTTCACCGCTTTCAACTTTG & qRT-PCR Amplicon Bd_Bahd_5 & 1.72 \\
\hline prHM12 & Antisense & TCGCAACCTGGTCTTTGACAC & qRT-PCR Amplicon Bd_Bahd_5 & \\
\hline prHM19 & Sense & CCGGTGCTAGCCCTGGAATA & qRT-PCR Amplicon Bd_Bahd_10 & 1.77 \\
\hline prHM20 & Antisense & TGCACGCGTTGTACTCCGA & qRT-PCR Amplicon Bd_Bahd_10 & \\
\hline prHM33 & Sense & CGCAAGACAATGACCGCTATG & qRT-PCR Reference gene Bd_UBC18 & 1.78 \\
\hline prHM34 & Antisense & CCAATCCGACGCCTCCTTATA & qRT-PCR Reference gene Bd_UBC18 & \\
\hline prHM35 & Sense & TGTTTGTGTCGGATTGGACGA & qRT-PCR Amplicon Bd_Bahd_7 & 1.69 \\
\hline prHM36 & Antisense & ACCGCCATGTAGTCCGCATAA & qRT-PCR Amplicon Bd_Bahd_7 & \\
\hline prHM43 & Sense & TTCTCGTATCACCCCTTCATGG & qRT-PCR Amplicon Bd_Bahd_9 & 1.75 \\
\hline prHM44 & Antisense & GGTGGTCTTCCTCCACACACAT & qRT-PCR Amplicon Bd_Bahd_9 & \\
\hline prHM55 & Sense & TGGCTTCTACGGCAACTGCTA & qRT-PCR Amplicon Bd_Bahd_2p1 & 1.79 \\
\hline prHM56 & Antisense & GCTTCCCGTCCTTGATGATCT & qRT-PCR Amplicon Bd_Bahd_2p1 & \\
\hline prHM61 & Sense & AAGCGGCTCGAGTACCTG & qRT-PCR Amplicon Bd_Bahd_2p2 & 1.82 \\
\hline prHM62 & Antisense & GCCATTGTTGCTGGAGTTGT & qRT-PCR Amplicon Bd_Bahd_2p2 & \\
\hline prHM65 & Sense & CCACGTCTGCTTCGCCATG & qRT-PCR Amplicon Bd_Bahd_8 & 1.79 \\
\hline prHM66 & Antisense & CGCATGATGTAGTAGCAGTTGCC & qRT-PCR Amplicon Bd_Bahd_8 & \\
\hline prHM69 & Sense & ATCCCGCCATCCAACATCTAC & qRT-PCR Amplicon Bd_Bahd_12 & 1.79 \\
\hline prHM70 & Antisense & CGGATCTGGCCTTGATGTTGT & qRT-PCR Amplicon Bd_Bahd_12 & \\
\hline prHM75 & Sense & TTCAACAGCATGGATGGCC & qRT-PCR Reference gene Bd_SDH & 1.76 \\
\hline prHM76 & Antisense & ATCTTCGGTTGCAGAGCTCCT & qRT-PCR Reference gene Bd_SDH & \\
\hline
\end{tabular}

Phytozome $^{1}$ containing the PFAM domain PF02458. An initial tree was generated and all sequences contained within the candidate group identified in Mitchell et al. (2007) were aligned with the MUSCLE algorithm (Edgar, 2004). Gapped columns were removed, followed by phylogeny analysis of aligned sequences in the PhyML package (Guindon et al., 2005) using the Whelan and Goldman (2001) model. An initial run optimized the gamma and invariant proportion parameters; these were then held constant for 100 runs for bootstrap non-parametric analysis and the maximum likelihood tree is presented.

\section{DETERMINATION OF TRANSCRIPT ABUNDANCE}

RNA was extracted using a cetyltrimethylammonium bromide (CTAB) method following Chang etal. (1993). Quantitative PCR was performed as in Pellny et al. (2008) on an Applied Biosystems 7500 real-time PCR system ${ }^{2}$ using SYBR green JumpStart Kit (Sigma-Aldrich ${ }^{3}$ ) following the manufacturer's instructions. The expression of the genes of interest was normalized

\footnotetext{
${ }^{1}$ www.phytozome.org

${ }^{2}$ www.appliedbiosystems.com

${ }^{3}$ www.sigmaaldrich.com
}

with two endogenous controls ubiquitin-conjugating enzyme 18 (UBC18) and succinate dehydrogenase (SDH). Putative house-keeping genes glyceraldehyde-3-phosphate dehydrogenase (GAPDH), S-adenosylmethionine decarboxylase (SAMDC), and peptide deformylase 2 (PDF2) were also tested but found less stable over the different tissue and time points. Expression calculations were performed as suggested by Rieu and Powers (2009). Individual amplification efficiencies were established with LinRegPCR using a window-of-linearity and the relative quantities (RQs) for the target genes were calculated. This was normalized with the geometrical mean of the RQs of the control genes. Primers used and average efficiency of pairs are listed in Table 2.

\section{DETERMINATION OF BOUND PHENOLIC CONTENT}

Cell wall bound phenolics were released by alkaline hydrolysis of alcohol insoluble residues (AIRs) from samples $(20 \mathrm{mg})$ of freezedried, ground tissue and extraction into ethyl acetate as previously described (Pellny et al., 2012). 3,5-Dichloro-4-hydroxybenzoic acid $\left(20 \mu \mathrm{L}\right.$ at $\left.1.5 \mathrm{mg} \mathrm{mL}^{-1}\right)$ was added to AIR of all samples, prior to alkaline hydrolysis, as an internal standard. Samples were dissolved in $1 \mathrm{~mL} \mathrm{50 \%} \mathrm{methanol:2 \%} \mathrm{acetic} \mathrm{acid} \mathrm{(v:v)} \mathrm{and} \mathrm{bound}$ phenolic acids from $40 \mu \mathrm{L}$ of extract separated by HPLC on a 
Shimadzu Prominence high-performance liquid chromatograph as described by Waldron et al. (1996) but using a binary gradient pump system. Quantitation of FA and $p$ CA was by integration of peak areas at $280 \mathrm{~nm}$ with reference to calibrations made using known amounts of pure compounds. Peaks of the major FA dimers were identified by comparison of retention times with pure standards kindly supplied by Professor John Ralph (Lu et al., 2012; 5-5, 8-O-4, 8-5-benzofuran) or by comparison of spectrum with that in Waldron et al. (1996) (8-5). Dimer quantitation was achieved relative to FA monomer using areas of these peaks and the response factors for dimers and FA monomer in Waldron et al. (1996). All samples were extracted and analyzed in triplicate.

\section{REFERENCES}

Akin, D. E. (2008). Plant cell wall aromatics: influence on degradation of biomass. Biofuels Bioprod. Biorefin. 2, 288-303.

Anders, N., Wilkinson, M. D., Lovegrove, A., Freeman, J., Tryfona, T., Pellny, T. K., et al. (2012). Glycosyl transferases in family 61 mediate arabinofuranosyl transfer onto xylan in grasses. Proc. Natl. Acad. Sci. U.S.A. 109, 989-993.

Baranowski, J. D., Davidson, P. M., Nagel, C. W., and Branen, A. L. (1980). Inhibition of Saccharomyces cerevisiae by naturally occurring hydroxycinnamates. J. Food Sci. 45, 592-594.

Bartley, L. E., Peck, M. L., Kim, S.R., Ebert, B., Maniseri, C., Chiniquy, D., et al. (2013). Overexpression of a BAHD acyltransferase, OsAt10, alters rice cell wall hydroxycinnamic acid content and saccharification. Plant Physiol. doi: 10.1104/pp.112.208694 [Epub ahead of print].

Buanafina, M. M. D. (2009). Feruloylation in grasses: current and future perspectives. Mol. Plant 2, 861-872.

Carpita, N. C. (1996). Structure and biogenesis of the cell walls of grasses. Annu. Rev. Plant Physiol. Plant Mol. Biol. 47, 445-476.

Chang, S. J., Puryea, J., and Cairney, J. (1993). A simple and efficient method for isolating RNA from pine tree. Plant Mol. Biol. Rep. 11, 113-116.

Christensen, U., Alonso-Simon, A., Scheller, H. V., Willats, W. G. T., and Harholt, J. (2010). Characterization of the primary cell walls of seedlings of Brachypodium distachyon - a potential model plant for temperate grasses. Phytochemistry 71, 62-69.

D'Auria, J. C. (2006). Acyltransferases in plants: a good time to be BAHD. Curr. Opin. Plant Biol. 9, 331-340.

Dhugga, K. S. (2007). Maize biomass yield and composition for biofuels. Crop Sci. 47, 22112227.
Edgar, R. C. (2004). MUSCLE: multiple sequence alignment with high accuracy and high through1797.

Grabber, J. H. (2005). How do lignin composition, structure, and crosslinking affect degradability? A review of cell wall model studies. Crop Sci. $45,820-831$.

Grabber, J. H., Ralph, J., Lapierre C., and Barriere, Y. (2004). Genetic and molecular basis of grass cellwall degradability. I. Lignin-cell wall matrix interactions. C. R. Biol. 327 455-465.

Guindon, S., Lethiec, F., Duroux, P., and Gascuel, O. (2005). PHYML Online - a web server for fast maximum likelihood-based phylogenetic inference. Nucleic Acids Res. 33, W557-W559.

Harris, P. J., and Trethewey, J. A. K. (2010). The distribution of esterlinked ferulic acid in the cell walls of angiosperms. Phytochem. Rev. 9, 19-33.

Ishii, T. (1991). Isolation and characterization of a diferuloyl arabinoxylan hexasaccharide from bamboo shoot cell-walls. Carbohydr. Res. 219, $15-22$.

Konishi, T., Takeda, T., Miyazaki, Y., Ohnishi-Kameyama, M., Hayashi, T., O’Neill, M. A., et al. (2007). A plant mutase that interconverts UDP-arabinofuranose and UDParabinopyranose. Glycobiology 17, 345-354.

Lu, F. C., Wei, L. P., Azarpira, A., and Ralph, J. (2012). Rapid syntheses of dehydrodiferulates via biomimetic radical coupling reactions of ethyl ferulate. J. Agric. Food Chem. 60, 8272-8277.

Luo, J., Nishiyama, Y., Fuell, C. Taguchi, G., Elliott, K., Hill, L., et al. (2007). Convergent evolution in the BAHD family of acyl transferases: identification and characterization of anthocyanin acyl transferases from Arabidopsis thaliana. Plant J. 50, 678-695. put. Nucleic Acids Res. 32, 1792-

\section{ACKNOWLEDGMENTS}

This work was supported by the UK Biotechnology and Biological Sciences Research Council (BBSRC), partly by a BBSRC Sustainable Bioenergy Centre (BSBEC) Biomass Programme Grant and we thank the PI, Dr Angela Karp (Rothamsted), for her support. Support for Hugo B. C. Molinari from BBSRC and funding from the UK Department of Environment, Food and Rural Affairs under a BSBEC-EMBRAPA partnership scheme is gratefully acknowledged. Funding for Till K. Pellny was from a BBSRC grant to Rowan A. C. Mitchell and Peter R. Shewry (BB/F014295/1). Rothamsted Research receives grant-aided support from the BBSRC.

Mastrangelo, L., Lenucci, M., Piro, G. and Dalessandro, G. (2009). Evidence for intra- and extra-protoplasmic feruloylation and cross-linking in wheat seedling roots. Planta 229, 343-355.

Mitchell, R. A. C., Dupree, P., and Shewry, P. R. (2007). A novel bioinformatics approach identifies candidate genes for the synthesis and feruloylation of arabinoxylan. Plant Physiol. 144, 43-53.

Myton, K. E., and Fry, S. C. (1994) Intraprotoplasmic feruloylation of arabinoxylans in Festuca arundinacea cell cultures. Planta 193, 326-330.

Pellny, T. K., Lovegrove, A., Freeman, J., Tosi, P., Love, C. G., Knox, J. P., et al. (2012). Cell walls of developing wheat starchy endosperm: comparison of composition and RNA-Seq transcriptome. Plant Physiol. 158, 612-627.

Pellny, T. K., Van Aken, O., Dutilleul, C., Wolff, T., Groten, K., Bor, M., et al. (2008). Mitochondrial respiratory pathways modulate nitrate sensing and nitrogen-dependent regulation of plant architecture in Nicotiana sylvestris. Plant J. 54, 976-992.

Piston, F., Uauy, C., Fu, L. H., Langston, J., Labavitch, J., and Dubcovsky, J. (2010). Down-regulation of four putative arabinoxylan feruloyl transferase genes from family PF02458 reduces ester-linked ferulate content in rice cell walls. Planta 231, 677-691.

Ralph, J., Grabber, J. H., and Hatfield, R. D. (1995). Lignin-ferulate crosslinks in grasses - active incorporation of ferulate polysaccharide esters into ryegrass lignins. Carbohydr. Res. 275, 167-178.

Rancour, D., Marita, J., and Hatfield, R. D. (2012). Cell wall composition throughout development for the model grass Brachypodium distachyon. Front. Plant Sci. 3:266. doi: 10.3389/fpls.2012.00266

Rautengarten, C., Ebert, B., Herter, T., Petzold, C. J., Ishii, T., Mukhopadhyay, A., et al. (2011). The interconversion of UDP-arabinopyranose and UDP-arabinofuranose is indispensable for plant development in Arabidopsis. Plant Cell 23, 1373 1390.

Rieu, I., and Powers, S. J. (2009). Realtime quantitative RT-PCR: design, calculations, and statistics. Plant Cell 21, 1031-1033.

Sato, Y., Namiki, N., Takehisa, H., Kamatsuki, K., Minami, H., Ikawa, H., et al. (2013). RiceFREND: a platform for retrieving coexpressed gene networks in rice. Nucleic Acids Res. 41, D1214-D1221.

Scheller, H. V., and Ulvskov, P. (2010). Hemicelluloses. Annu. Rev. Plant Biol. 61, 263-289.

Shewry, P. R., Freeman, J., Wilkinson, M. D., Pellny, T. K., and Mitchell, R. A. C. (2011). "Challenges and opportunities for using wheat for biofuel production," in Energy Crops, eds A. Karp and N. G. Halford (Cambridge: RSC Publishing), 13-26.

Vogel, J. P., Garvin, D. F., Mockler, T. C., Schmutz, J., Rokhsar, D., Bevan, M. W., etal. (2010). Genome sequencing and analysis of the model grass Brachypodium distachyon. Nature 463, 763-768.

Waldron, K. W., Parr, A. J., Ng, A., and Ralph, J. (1996). Cell wall esterified phenolic dimers: identification and quantification by reverse phase high performance liquid chromatography and diode array detection. Phytochem. Anal. 7, 305-312.

Whelan, S., and Goldman, N. (2001). A general empirical model of protein evolution derived from multiple protein families using a maximumlikelihood approach. Mol. Biol. Evol. 18, 691-699.

Withers, S., Lu, F. C., Kim, H., Zhu, Y. M., Ralph, J., and Wilkerson, C. G. (2012). Identification of grassspecific enzyme that acylates monolignols with $p$-coumarate. J. Biol. Chem. 287, 8347-8355.

Yoshida-Shimokawa, T., Yoshida, S., Kakegawa, K., and Ishii, T. (2001). Enzymic feruloylation of arabinoxy- 
lan-trisaccharide by feruloyl-CoA: arabinoxylan-trisaccharide $O$-hydroxycinnamoyl transferase from Oryza sativa. Planta 212, 470-474.

Conflict of Interest Statement: The authors declare that the research was conducted in the absence of any commercial or financial relationships that could be construed as a potential conflict of interest.

Received: 14 December 2012; accepted: 25 February 2013; published online: 15 March 2013.

Citation: Molinari HBC, Pellny TK, Freeman J, Shewry $P R$ and Mitchell RAC (2013) Grass cell wall feruloylation: distribution of bound ferulate and candidate gene expression in Brachypodium distachyon. Front. Plant Sci. 4:50. doi: 10.3389/fpls.2013.00050

This article was submitted to Frontiers in Plant Biotechnology, a specialty of Frontiers in Plant Science.

Copyright (c) 2013 Molinari, Pellny, Freeman, Shewry and Mitchell.
This is an open-access article distributed under the terms of the Creative Commons Attribution License, which permits use, distribution and reproduction in other forums, provided the original authors and source are credited and subject to any copyright notices concerning any third-party graphics etc. 